\title{
Effect of trailing edge shape on hydrodynamic damping for a hydrofoil
}

\author{
Zhifeng Yao ${ }^{a}$, Fujun Wang ${ }^{\mathrm{a}, *}$, Matthieu Dreyer ${ }^{\mathrm{b}}$, Mohamed Farhat ${ }^{\mathrm{b}}$ \\ a College of Water Resources and Civil Engineering, China Agricultural University, Beijing, China \\ ${ }^{\mathrm{b}}$ Laboratory for Hydraulic Machines, Swiss Federal Institute of Technology, Lausanne, Switzerland
}

\section{A R T I C L E I N F O}

\section{Article history:}

Received 27 August 2013

Accepted 3 September 2014

\section{Keywords:}

Hydrofoil

Hydrodynamic damping

Trailing edge shape

Donaldson

\begin{abstract}
A B S T R A C T
Flow induced vibration on a hydrofoil may be significantly reduced with a slight modification of the trailing edge without alteration of the hydrodynamic performance. Particularly, the so called Donaldson trailing edge shape gave remarkable results and is being used in a variety of industrial applications. Nevertheless, the physics behind vibration reduction is still not understood. In the present study, we have investigated the hydrodynamic damping of a 2D hydrofoil with Donaldson trailing edge shape. The results are compared with the same hydrofoil with blunt trailing edge. The tests are carried out in EPFL high speed cavitation tunnel and two piezoelectric patches are used for the hydrofoil excitation in non-intrusive way. It was found that the hydrodynamic damping is significantly increased with the Donaldson cut. Besides, as the flow velocity is increased, the hydrodynamic damping is found to remain almost constant up to the hydrofoil resonance and then increases linearly, for both tested trailing edge shapes and for both first bending and torsion modes.
\end{abstract}

(c) 2014 Elsevier Ltd. All rights reserved.

\section{Introduction}

Flow induced vibration is a major issue in a variety of industrial applications. In the particular case of hydropower generation, the strong increase in global energy demand and the liberalization of electricity market is putting more pressure on designers of turbines and pumps. Hydropower sector is struggling to supply more energy with enhanced efficiency and security while playing a growing role in stabilizing the electricity grid. In this context, hydraulic machines are operated more often at off-design conditions and subjected to frequent starts and stops, which may lead to excessive vibration with increased risks of mechanical failures.

Among the various hydrodynamic excitation sources of vibration, the vortex shedding from hydrofoils trailing edge is particularly dangerous because of the strong coherence of alternate shedding, which generates lift fluctuation and may lead to mechanical resonances. Although the phenomenon of vortex shedding has been widely investigated, most of the studies were limited to the case of the flow around a cylinder at relatively low Reynolds number (Williamson and Govardhan, 2004). Bourgoyne et al. (2003) carried out time-averaged flow-field measurements for a modified NACA 16 with two trailing-edge bevel angles at high Reynolds number (between $1 \times 10^{6}$ and $50 \times 10^{6}$ ). A strong dependence on Reynolds number was revealed, which the authors explained by a change in flow's dynamic components. Shannon (2007) investigated the mechanism of trailing edge noise by measuring flow field and acoustic variables for incompressible and high Reynolds

\footnotetext{
* Corresponding author.

E-mail addresses: yzf@cau.edu.cn (Z. Yao), wangfj@cau.edu.cn (F. Wang).
} 


\begin{tabular}{|c|c|c|c|}
\hline \multicolumn{2}{|c|}{ Nomenclature } & \multirow{2}{*}{$\begin{array}{l}f \\
f_{\mathrm{e}} \\
f_{1}\end{array}$} & \multirow{2}{*}{$\begin{array}{l}\text { hydrofoil vibration frequency [Hz] } \\
\text { excitation frequency [Hz] } \\
\text { undamped natural frequency for } 1 \text { st bending } \\
\text { mode }[\mathrm{Hz}]\end{array}$} \\
\hline$\beta$ & $\begin{array}{l}\text { ratio of the excitation frequency to the } \\
\text { undamped natural frequency [dimensionless] }\end{array}$ & & \\
\hline$\varsigma$ & total damping factor [dimensionless] & $f_{2}$ & undamped natural frequency for 1 st torsional \\
\hline$\varsigma_{\mathrm{s}}$ & structure damping factor [dimensionless] & & mode $[\mathrm{Hz}]$ \\
\hline 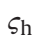 & hydrodynamic damping factor [dimensionless] & $H$ & hydrofoil trailing edge thickness [m] \\
\hline$\mu$ & dynamic amplification factor [dimensionless] & $L$ & the profile chord length $[\mathrm{m}]$ \\
\hline$\nu$ & Poisson's ratio [dimensionless] & $x$ & deflection of the patch $[\mathrm{m}]$ \\
\hline$\rho_{\mathrm{s}}$ & density $\left[\mathrm{kg} / \mathrm{m}^{3}\right]$ & $x_{0}$ & static deflection [m] \\
\hline$v$ & kinematic viscosity $\left[\mathrm{m}^{2} / \mathrm{s}\right]$ & $\dot{x}$ & velocity [m/s] \\
\hline$C^{*}$ & reduced flow velocity $C^{*}=C / f L$ & $\ddot{x}$ & acceleration $\left[\mathrm{m} / \mathrm{s}^{2}\right]$ \\
\hline & [dimensionless] & $w_{\mathrm{n}}$ & natural frequency of the hydrofoil [rad/s] \\
\hline C & flow velocity $[\mathrm{m} / \mathrm{s}]$ & $\mathrm{S}_{\mathrm{t}}$ & Strouhal number $S_{t}=f h / C$ [dimensionless] \\
\hline E & material Young modulus [GPa] & $\mathrm{R}_{\mathrm{e}}$ & Reynolds number $\mathrm{R}_{\mathrm{e}}=\mathrm{Ch} / v$ [dimensionless] \\
\hline
\end{tabular}

number flows. Ausoni et al. (2007) have shown how a blunt trailing edge hydrofoil may undergo large vibration when the shedding frequency locks onto the resonance frequency of its 1st torsion mode. They have also highlighted the significant role of the boundary layer and cavitation occurrence on the roll-up mechanism of trailing edge vortices.

In an attempt to mitigate the flow induced vibration due to wake instability, a large number of remedies were proposed, with a common idea of disturbing the process of vortex roll-up in the formation zone. Theoretical analysis of trailing edge noise produced by turbulent flow over an airfoil was presented by Howe (1988). He concluded that surface beveling had a significant effect on the radiation only at sufficiently high frequencies that the trailing edge may be regarded as a straightsided wedge over distances of the order of the turbulence length scale. Krentel and Nitsche (2013) performed experimental investigations on four different trailing edge shapes of a bluff airfoil, in order to mitigate the process of periodic and alternate vortex shedding. They have shown that a significant reduction of noise may be obtained with optimized trailing edge geometry. In hydraulic machines, it is well known that vortex shedding from the blades trailing edge may excite the mechanical structure. By sharpening the trailing edge, the frequency of the noise may be shifted with reduced amplitude (Ruprecht et al., 2003). Mosallem (2008) performed numerical simulation of the flow field past beveled trailing edges. The results displayed an asymmetric wake beyond $27^{\circ}$ beveled trailing edge and Von Kàrman street vortices beyond $60^{\circ}$ beveled trailing edge. In a well detailed experiment, Zobeiri et al. (2012) has shown how a simple $30^{\circ}$ oblique truncation of a hydrofoil trailing edge may significantly reduce the vibration without alteration of hydrodynamic performances. They clearly observed that the oblique truncation generates a phase shift between upper and lower vortices and leads to their "collision" and partial cancellation. As a result, a significant thickening of their viscous cores and a reduction of their intensity were obtained. Donaldson (1956) performed systematic measurements of flow-induced vibration in Francis turbine runners with a large variety of trailing edge shapes. He found a substantial reduction of vibration with a combination of a straight line at $45^{\circ}$ angle and a 3rd polynomial curve (see Fig. 1). Although this so-called Donaldson trailing edge shape performs well in a variety of industrial applications, the physics behind the vibration reduction is still lacking.

One way to evaluate the effect of trailing edge shape on the flow induced vibration is the measurement of resulting change in the hydrodynamic damping. In fact, any alteration of the wake dynamic directly impacts the fluid structure interaction and the way the flow reacts to damp out the structural vibration. Hydrodynamic damping has long been of special interest in many flow configurations such as cables, pipes (Blevins, 1990; Naudascher and Rockwell, 2006) and cylinders (Chaplin and Subbiah, 1998). Kaminer and Nastenko (1976) first measured the hydrodynamic damping related to bending oscillations of blade profiles in water flow. He pointed out that hydrodynamic damping varies in a linear way with reduced flow velocity and may be influenced by the profile solidity. Recently, Seeley et al. (2012) investigated experimentally the hydrodynamic damping of three hydrofoils. The results indicated that although the natural frequency was not altered by the flow, the damping ratio increased in a linear manner with respect to flow velocity.

The identification of a blade hydrodynamic damping is a difficult task as previously reported by many authors (Seeley et al., 2012; Roth et al., 2009). The vibration due to flowing water makes traditional testing methods of dynamic system identification impractical. In order to overcome these difficulties, Roth et al. (2009) used underwater spark generated bubbles to create shock waves that excite the hydrofoil in a wide frequency band in non-intrusive way. The mechanical response was monitored on the hydrofoil surface with a laser vibrometer. The damping factor was estimated by fitting an exponential function on the envelope of the hydrofoil impulse response. Nevertheless, it was found that beyond a threshold value of $15 \mathrm{~m} / \mathrm{s}$ flow velocity, such a method is no more appropriate since the flow induced vibration becomes significantly larger than the impulse response. An alternate technique for the mechanical excitation of a hydrofoil is the use of embedded piezoelectric actuators (Seeley et al., 2012; De La Torre, 2013; De La Torre et al., 2013), which provides an interesting and non-intrusive way to excite a specific eigen mode of immersed hydrofoils. 
(a)

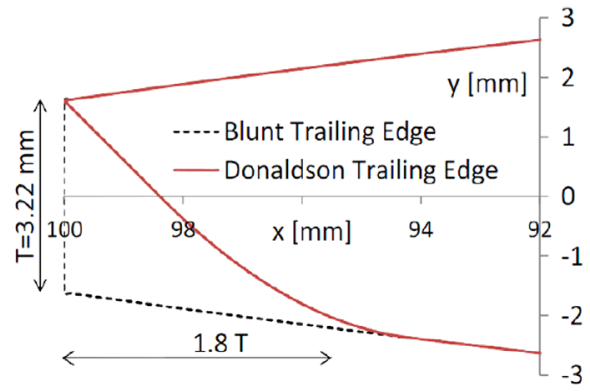

(b)

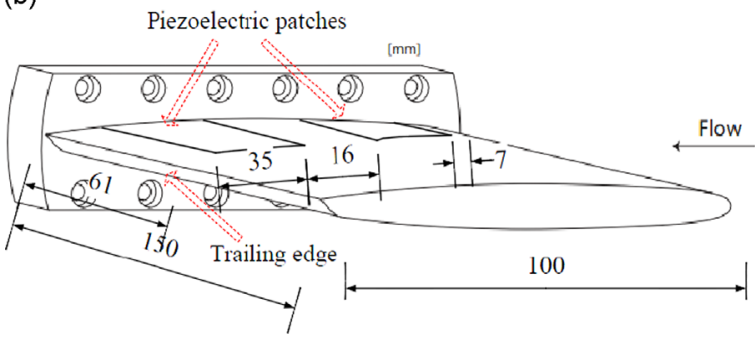

Fig. 1. Two tested tailing edge shapes: Truncated and Donaldson trailing edges (a); NACA 0009 hydrofoil embedded with piezoelectric patches (b).

In the present paper, we intend to evaluate experimentally the effect of Donaldson trailing edge shape on the hydrodynamic damping of a symmetric hydrofoil at high Reynolds number with the help of two embedded piezoelectric patches. The hydrofoil is placed in the test section of EPFL high speed cavitation tunnel with the possibility to vary the flow velocity in a wide range. The results are compared to a similar hydrofoil with blunt trailing edge.

\section{Experimental setup and methods}

\subsection{The case study}

The experimental investigations are carried out in the EPFL high-speed cavitation tunnel (Avellan et al., 1987), shown in Fig. 2. The test hydrofoil is mounted in the middle of $150 \times 150 \times 750 \mathrm{~mm}^{3}$ test section, with maximum inlet velocity of $50 \mathrm{~m} / \mathrm{s}$. The static pressure may be increased up to $1.6 \mathrm{MPa}$. The free stream turbulence intensity is around $1 \%$.

The tested hydrofoil is a NACA 0009 with $100 \mathrm{~mm}$ chord length and $150 \mathrm{~mm}$ span. The baseline geometry is given in Eq. (1) and Eq. (2) where $x$ and $y$ denote stream wise and transverse coordinates. The maximum thickness is $10 \mathrm{~mm}$ and is located at mid-chord. As illustrated in Fig. 1, the blunt trailing edge is replaced by a Donaldson cut, which is a combination of $45^{\circ}$ straight line and a 3rd polynomial curve $y=-1.332+51.142 x-637.51 x^{2}+2595.18 x^{3}$. The hydrofoil is made of aluminum with Young modulus $E=69 \mathrm{GPa}$, density $\rho_{\mathrm{s}}=2700 \mathrm{~kg} / \mathrm{m}^{3}$ and Poisson's ratio $\nu=0.334$. The hydrofoil is mounted in the test section with perfect embedding on one side while the other side is left free to vibrate with $0.1 \mathrm{~mm}$ gap.

$$
\begin{array}{ll}
0 \leq \frac{y}{L} \leq 0.5 & \frac{y}{L}=0.1737\left(\frac{x}{L}\right)^{1 / 2}-0.2422\left(\frac{x}{L}\right)+0.3046\left(\frac{x}{L}\right)^{2}-0.26577\left(\frac{x}{L}\right)^{3}, \\
0.5 \leq \frac{y}{L} \leq 1 & \frac{y}{L}=0.0004+0.1737\left(1-\frac{x}{L}\right)-0.1898\left(1-\frac{x}{L}\right)^{2}+0.0387\left(1-\frac{x}{L}\right)^{3} .
\end{array}
$$

Two PZT patches (P-876.A 12) made of piezoelectric ceramic material based on modified lead zirconate titanate are fitted on the hydrofoil surface and allow for either mechanical excitation or vibration measurements (see Fig. 1). The patches are $61 \mathrm{~mm}$ long, $35 \mathrm{~mm}$ wide and $0.5 \mathrm{~mm}$ thick. Thin cavities were machined on the hydrofoil suction side to embed the patches with no alteration of the hydrofoil geometry. Tiny groves were also machined on the hydrofoil surface for wires path. The patches and the cables were electrically isolated with a layer of epoxy. The use of piezoelectric patches for mechanical excitation of similar hydrofoil was previously tested and validated by De La Torre et al. (2012, 2013) and De La Torre (2013) in air as well as in still and flowing water. The use of the patch as a vibration sensor was also validated by comparing its output signal with a reference accelerometer. In our experiment, the upstream patch (close to the leading edge) was used to excite the hydrofoil. A voltage is fed to the metalized surface surrounding the ceramic material. The resulting contraction-expansion of the patch in a direction perpendicular to the electric field exerts a force on the substrate. A function generator (WAVETEK) is used to produce a $10 \mathrm{~V}$ amplitude sine wave, which is amplified 25 times before being applied to the patches. The downstream patch located near the trailing edge is used to measure the hydrofoil response. The signals are recorded using a NI PXI-4472 digitizer at $10 \mathrm{kHz}$ sampling frequency. 
(a)

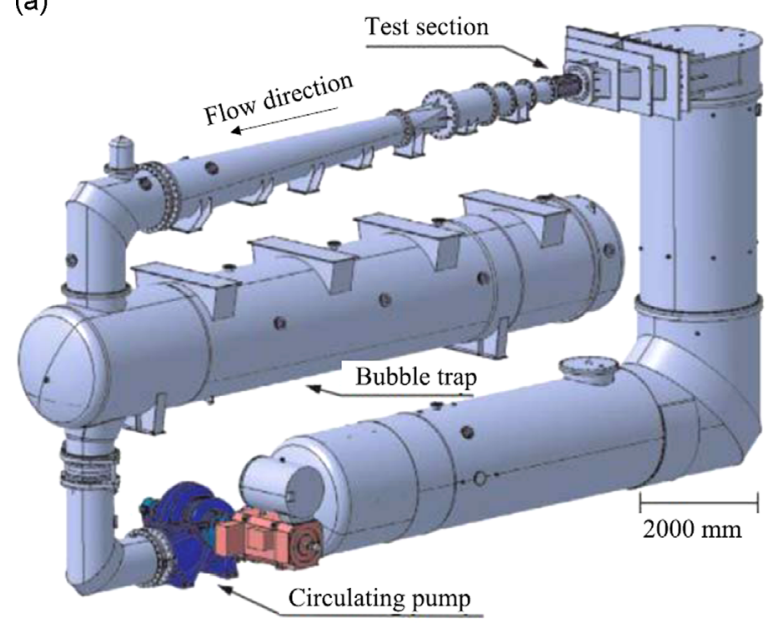

(b)

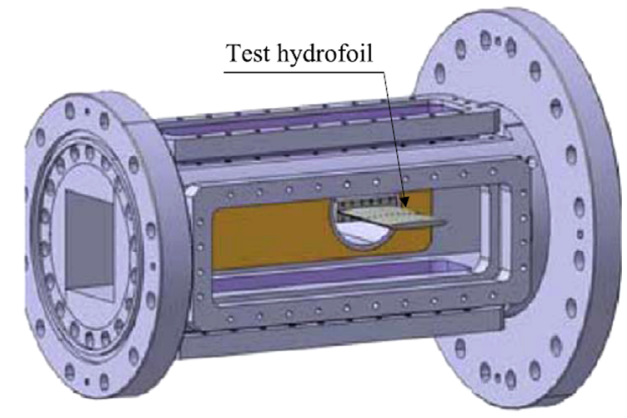

Fig. 2. EPFL-LMH high speed cavitation tunnel: hydraulic circuit (a) and test section (b).

\subsection{Theoretical background}

For the present measurements, the tested hydrofoil is always locked in to a particular mode, and the amplitude of the 1st torsion mode frequency is much higher than that of the 1st bending frequency. In these testing situations, the hydrofoil motion under harmonic loading may be modeled by a Single Degree of Freedom linear system (Kaminer and Nastenko, 1976; Seeley et al., 2012). According to the classical analytical approach, the structure displacement, $x$, measured by the patch close to hydrofoil trailing edge is governed by the following differential equation:

$$
\ddot{x}+2 \varsigma w_{n} \dot{x}+w_{n}^{2} x=\frac{F(t)}{m},
$$

where $x$ is the structure displacement, $\dot{x}$ is the velocity, and $\ddot{x}$ the acceleration. $\varsigma$ is the total damping factor, $m$ is the mass and $w_{n}$ is the natural frequency of the hydrofoil in water. $F(t)$ is the harmonic excitation force given by

$$
F(t)=F_{0} \sin \left(2 \pi f_{\mathrm{e}} t\right) \text {. }
$$

It is assumed that the structural damping $\left(\varsigma_{\mathrm{s}}\right)$ is negligible compared to hydrodynamic damping $\left(\zeta-\zeta_{\mathrm{h}}\right)$. It should be noticed that the hydrodynamic damping of a similar hydrofoil made of stainless steel with truncated trailing edge shown in Fig. 1(a) has already been measured in the same cavitation tunnel by Roth et al. (2009). The results are used here as a reference to compare the effects of the tailing edge shape on hydrodynamic damping.

The hydrodynamic damping is expected to lie between 0 and 1 . The so called dynamic amplification factor, $\mu$, of a single degree of freedom system is given by

$$
\mu=\frac{x}{x_{0}}=\frac{1}{\sqrt{\left(1-\beta^{2}\right)^{2}+(2 \varsigma \beta)^{2}}},
$$

where $x_{0}$ is the static deflection, and $\beta$ is the ratio of the excitation frequency $\left(f_{\mathrm{e}}\right)$ to the undamped natural frequency $\left(f_{n}\right)$ :

$$
\beta=\frac{f_{\mathrm{e}}}{f_{n}}=2 \pi \frac{f_{\mathrm{e}}}{w_{n}} .
$$

The reduced flow velocity $C^{*}$ is defined for a given vibration frequency, $f$, as the ratio of one vibration period $(1 / f)$ and the transit time for a fluid particle traveling from the leading edge to the trailing edge $(C / L)$ :

$$
C^{*}=\frac{C}{f L},
$$

where $C$ is the flow velocity and $L$ is the chord length.

\subsection{Eigen modes identification}

We have used the commercial code Ansys Mechanical APDL 13.0 to simulate the most relevant mode shapes and compute their corresponding eigen frequencies. We focused on the first bending and torsion modes as well as the second bending mode (see Fig. 3). In our experiment, we have applied a sine sweep excitation signal to the hydrofoil mounted in the test section in air as well as in still and flowing water. To this end, the harmonic excitation frequency was linearly increased from $10 \mathrm{~Hz}$ to $3000 \mathrm{~Hz}$ during a period of $5 \mathrm{~s}$. The voltage applied to the patch next to the leading edge varied from $-200 \mathrm{~V}$ 
(a)

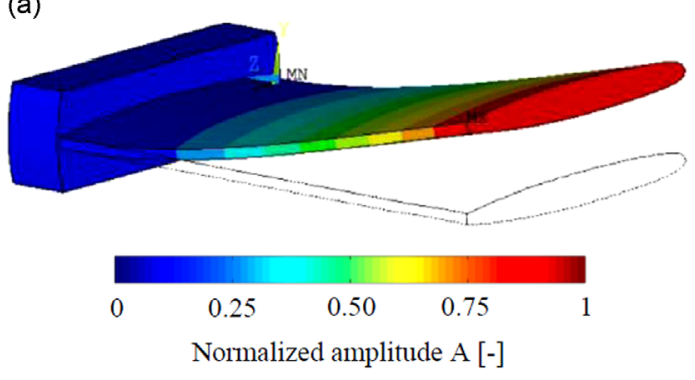

(b)

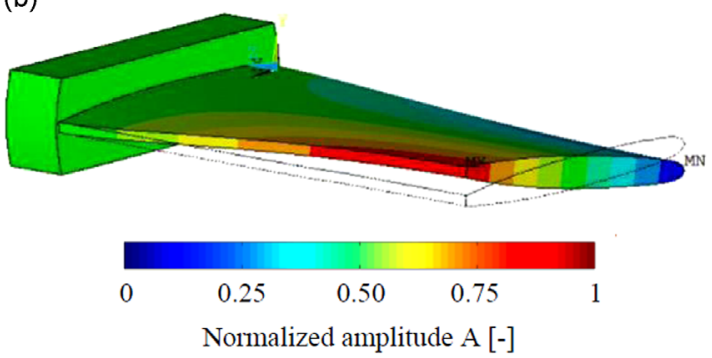

(c)

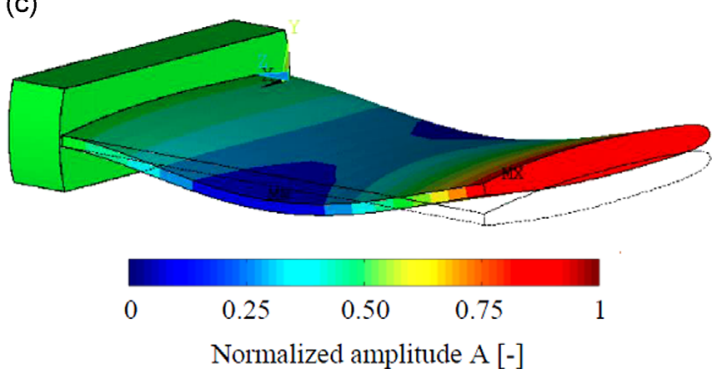

Fig. 3. Illustration of the first 3 eigen modes: 1 st bending (a), 1st torsion (b) and 2nd bending (c).

Table 1

Eigen frequencies (in $\mathrm{Hz}$ ) for Donaldson trailing edge hydrofoil.

\begin{tabular}{llclll}
\hline Mode & $\begin{array}{l}\text { In air } \\
\text { Experimental }\end{array}$ & $\begin{array}{l}\text { In air } \\
\text { Numerical }\end{array}$ & $\begin{array}{l}\text { Error } \\
(\%)\end{array}$ & $\begin{array}{l}\text { In still water } \\
\text { Experimental }\end{array}$ & $\begin{array}{l}\text { In still water } \\
\text { Numerical }\end{array}$ \\
\hline 1st Bending mode & 288.2 & 283 & 1.8 & 123.8 & $\begin{array}{l}\text { Error } \\
(\%)\end{array}$ \\
1st Torsion mode & 1027.6 & 1044 & 1.6 & 621.3 & 6.6 \\
2nd Bending mode & 1671.7 & 1715 & 2.6 & 900.0 & 637 \\
\hline
\end{tabular}

Table 2

Eigen frequencies (in Hz) for a blunt trailing edge hydrofoil (De La Torre, 2013).

\begin{tabular}{llllll}
\hline Mode & $\begin{array}{l}\text { In air } \\
\text { Experimental }\end{array}$ & $\begin{array}{l}\text { In air } \\
\text { Numerical }\end{array}$ & $\begin{array}{l}\text { Error } \\
(\%)\end{array}$ & $\begin{array}{l}\text { In still water } \\
\text { Experimental }\end{array}$ & $\begin{array}{l}\text { In still water } \\
\text { Numerical }\end{array}$ \\
\hline 1st Bending mode & 270.2 & 275 & 1.7 & 130.2 & $\begin{array}{l}\text { Error } \\
(\%)\end{array}$ \\
1st Torsion mode & 1018.6 & 1024 & 0.5 & 614.8 & 125.3 \\
2nd Bending mode & 1671 & 1679 & 0.5 & 886 & 630 \\
\hline
\end{tabular}

to $+400 \mathrm{~V}$. The response signal of the tailing edge patch is analyzed in spectral domain. Resonance frequencies were clearly revealed and associated to their corresponding mode shapes. The data collected for the Donaldson trailing edge are summarized in Table 1. We have also presented in Table 2 the results obtained by De La Torre (2013) on the same hydrofoil with blunt trailing edge. The computed values for resonance frequencies are found close to the measured ones. The maximum deviation (error) between experimental and numerical results is below 7\%. We may also observe that, as expected, the Donaldson truncation has almost no effect on the lower eigen frequencies. 


\subsection{Experimental procedure for hydrodynamic damping measurements}

Once the eigen frequencies of the first bending and torsion modes are determined, harmonic excitations of the hydrofoil are performed at different frequencies within a narrow band around the resonance frequencies. The tests are carried out for flow velocities ranging from 0 to $21 \mathrm{~m} / \mathrm{s}$. The incidence angle was kept equal to zero. The pressure at the test section inlet was maintained around $2.5 \times 10^{5}$ Pa to ensure cavitation free conditions.

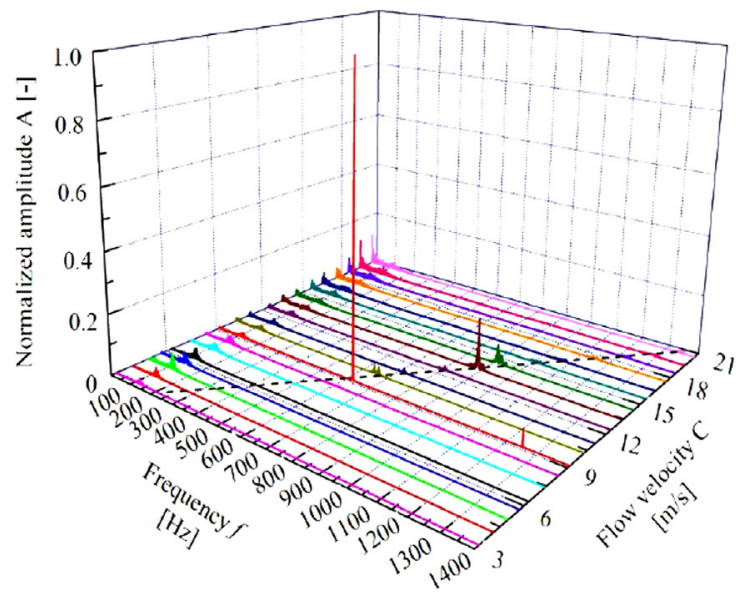

Fig. 4. Waterfall spectra of flow induced vibration of Donaldson trailing edge hydrofoil at different flow velocities.

(a)

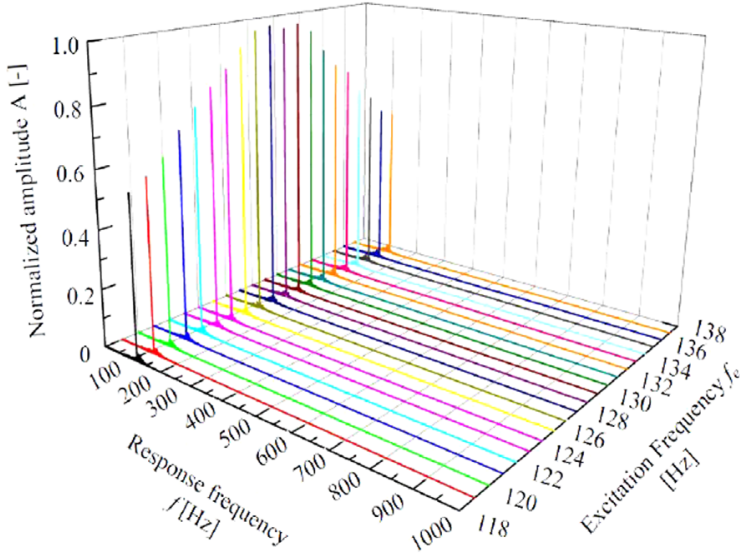

(b)

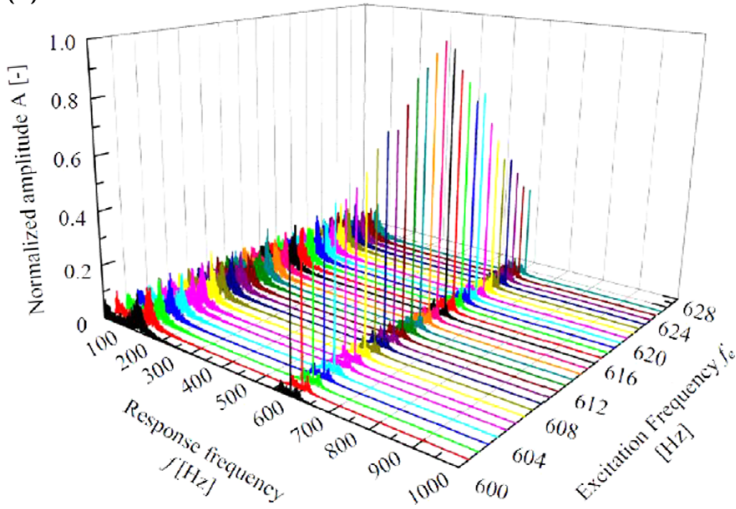

Fig. 5. Vibration of Donaldson tailing edge hydrofoil for different excitation frequencies at $7.03 \mathrm{~m} / \mathrm{s}$ flow velocity (a) and $9.45 \mathrm{~m} / \mathrm{s}$ flow velocity (b) The excited mode shapes are the first bending (a) and the first torsion (b). 
For a given flow conditions, harmonic excitation with an increasing frequency is used to excite the hydrofoil. At each excitation frequency, the hydrofoil response is recorded over $50 \mathrm{~s}$ duration at $10 \mathrm{kHz}$ sampling frequency. The signal is then divided into 10 segments to allow for averaging of RMS spectra. The amplitude of the vibration response is then extracted and plotted vs the excitation frequency. A curve fit is used to determine the best values of $f_{n}, \varsigma$, and $x_{0}$ according to Eq. (5) to match the experimental data (Seeley et al., 2012).

\section{Results and discussions}

We have presented in Fig. 4 the waterfall spectra of the flow induced vibration for Donaldson trailing edge, monitored by the trailing edge patch for flow velocities and Reynolds number ranging from $3 \mathrm{~m} / \mathrm{s}$ to $21 \mathrm{~m} / \mathrm{s}$, and from $0.3 \times 10^{6}$ to $2.1 \times 10^{6}$ respectively. The amplitude is normalized by the maximum value reached at $9.17 \mathrm{~m} / \mathrm{s}$. These spectra clearly show how the first bending mode is slightly excited at all tested flow velocities with no visible resonance. Besides, they also reveal the shedding frequency of Von Kàrman vortices, which increases linearly with the upstream velocity (dotted line in Fig. 4). The hydrofoil resonance occurs when the vortex shedding frequency approaches one of its natural frequencies. The vibration spectra reveal two resonances at $9.17 \mathrm{~m} / \mathrm{s}$ and $13.44 \mathrm{~m} / \mathrm{s}$, corresponding to the first torsion mode $(625 \mathrm{~Hz})$ and the second bending mode $(900 \mathrm{~Hz})$. Surprisingly, when compared with similar tests conducted on to the blunt trailing edge hydrofoil by Ausoni et al. (2007), Roth et al. (2009) and Zobeiri (2012), we do not observe any "lock-in" phenomenon for both mode shapes. This is another illustration of how the Donaldson trailing edge outperforms the blunt trailing edge. It is likely due to the significant change in the wake structure and a possible increase of the hydrodynamic damping, which limits the vibration amplitude of the hydrofoil and prevents from lock-in.

We have illustrated in Fig. 5 the averaged spectra of the hydrofoil vibration for Donaldson trailing edge, monitored by the trailing edge patch for upstream velocities of $7.03 \mathrm{~m} / \mathrm{s}$ and $9.45 \mathrm{~m} / \mathrm{s}$. For these configurations, the $1 \mathrm{st}$ bending and $1 \mathrm{st}$ torsion modes are respectively excited by the vortex shedding. The amplitudes are normalized by the maximum amplitude reached for the mode shape. The hydrofoil is excited by the harmonic signal. We may easily observe that the forced response of the hydrofoil is several orders larger than the flow induced vibration amplitude. This remains valid in the whole tested velocity
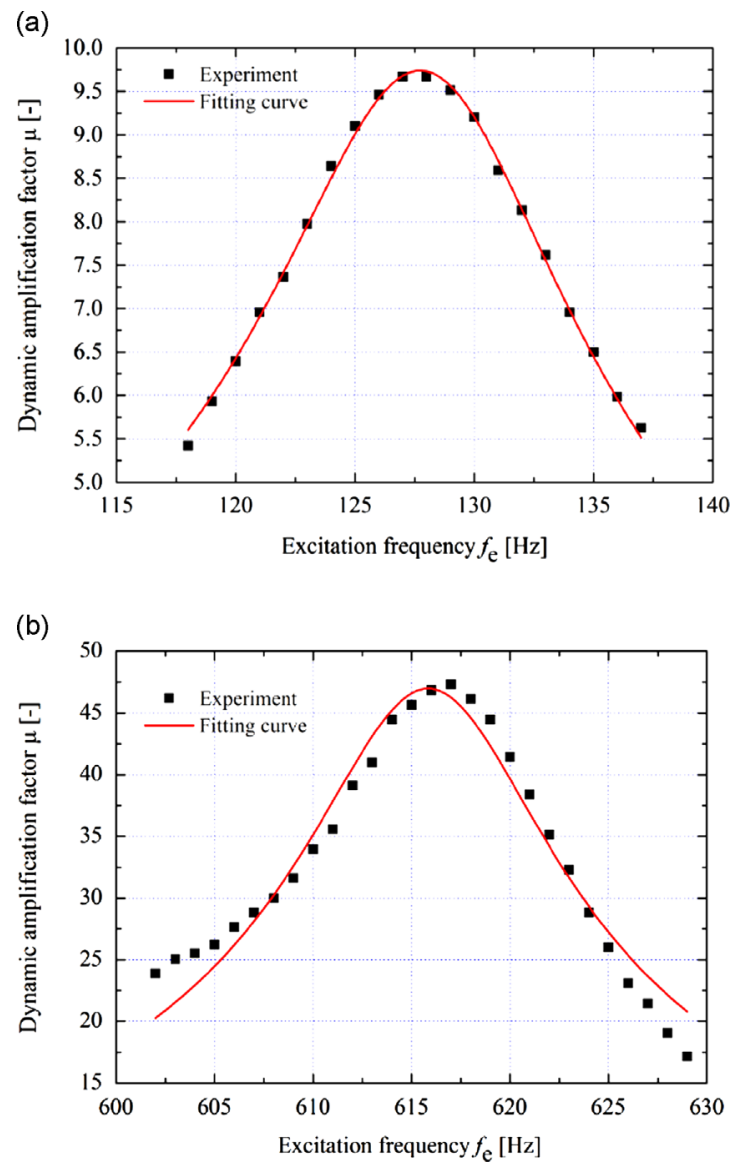

Fig. 6. The frequency response to harmonic loading and fitting curves. $7.03 \mathrm{~m} / \mathrm{s}$ flow velocity, yielding $f_{1}=128 \mathrm{~Hz}$ and $\zeta=0.051$ for bending mode (a) and $9.45 \mathrm{~m} / \mathrm{s}$ flow velocity, yielding $f_{2}=616 \mathrm{~Hz}$ and $\zeta=0.011$ for torsion mode (b). 
range, which makes the excitation procedure appropriate for the investigation of fluid-structure interaction in flowing water. Fig. 5 also reveals how the excitation signal with an increasing frequency allows for clear identification of the maximum hydrofoil response and a fine determination of the eigen frequencies in flowing water.

The response of the hydrofoil to harmonic excitations is processed and illustrated in Fig. 6. The dynamic amplification factor is plotted as a function of the excitation frequency for the first bending and torsion modes. Using Eq. (5), the best curves that fit experimental data are computed and plotted on the same figure. A perfect fitting curve may be observed for the 1st bending mode, in line with single degree of freedom assumption. For the 1st torsion mode, the experimental curve exhibits a non-symmetric shape with a fair agreement with the theoretical model near the resonance frequency and a significant discrepancy in the lower and higher ends of the frequency range. This may be explained by the fact that when the hydrofoil is resonating at its 1 st torsion mode, it also responds to less extent at the 1st bending mode as shown in Figs. 4 and 5(b). As a result, the coupling between the two modes reduces (resp. increases) the damping slightly below (resp. above) the torsion resonance frequency.

The hydrodynamic effect on added mass is illustrated in Fig. 7 where the natural frequencies of both the first bending and torsion modes are plotted as a function of the reduced velocity. A slight increase of the natural frequencies is clearly observed for both vibration modes when the reduced velocity is increased. For the torsion mode, the deviation of the experimental data from the straight line is likely due to the Karman vortices, which excite the hydrofoil at its 1st torsion mode.

The values of hydrodynamic damping for the first bending and torsion modes, derived from experimental data in still water and flowing water, are plotted in Fig. 8(a) as functions of reduced velocity. We have superposed on the same figures the results obtained formerly by Roth et al. (2009) for the blunt trailing edge hydrofoil.

For the 1st torsion mode, as the reduced velocity is increased, we may clearly observe two different trends of the hydrodynamic damping behavior. The graph reveals a threshold value of reduced velocity of about 0.15 , below which the hydrodynamic damping remains almost constant and above which it increases linearly. This threshold value corresponds to the resonance of the hydrofoil at its 1st torsion mode, sustained by the detachment of Kàrman vortices in its wake.

For the 1st bending mode, a similar behavior may be observed for the hydrodynamic damping in Fig. 8(a). Nevertheless, the sudden change from constant to linear trend is less obvious because of the lack of experimental data at low velocities. In fact, the minimum flow velocity allowed by the high speed cavitation tunnel is $3 \mathrm{~m} / \mathrm{s}$, while a flow velocity of $2 \mathrm{~m} / \mathrm{s}$ is
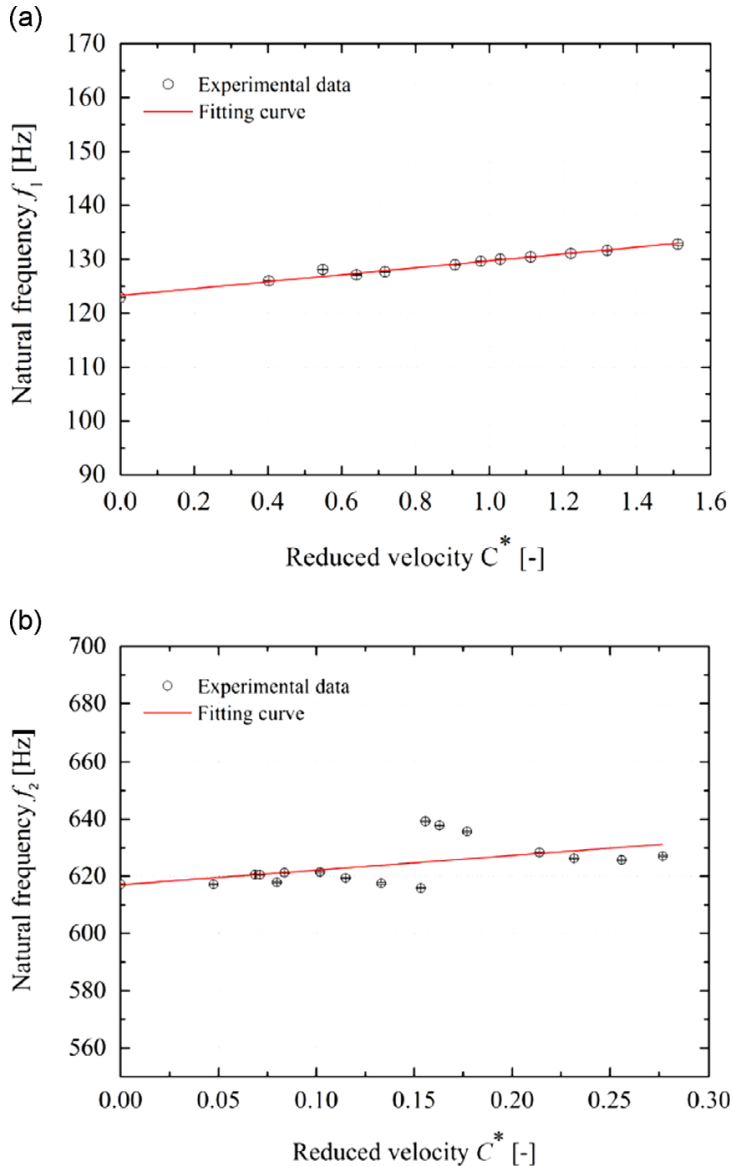

Fig. 7. The natural frequency of the first bending mode (a) and the first torsion mode (b) vs reduced velocity for Donaldson trailing edge. 

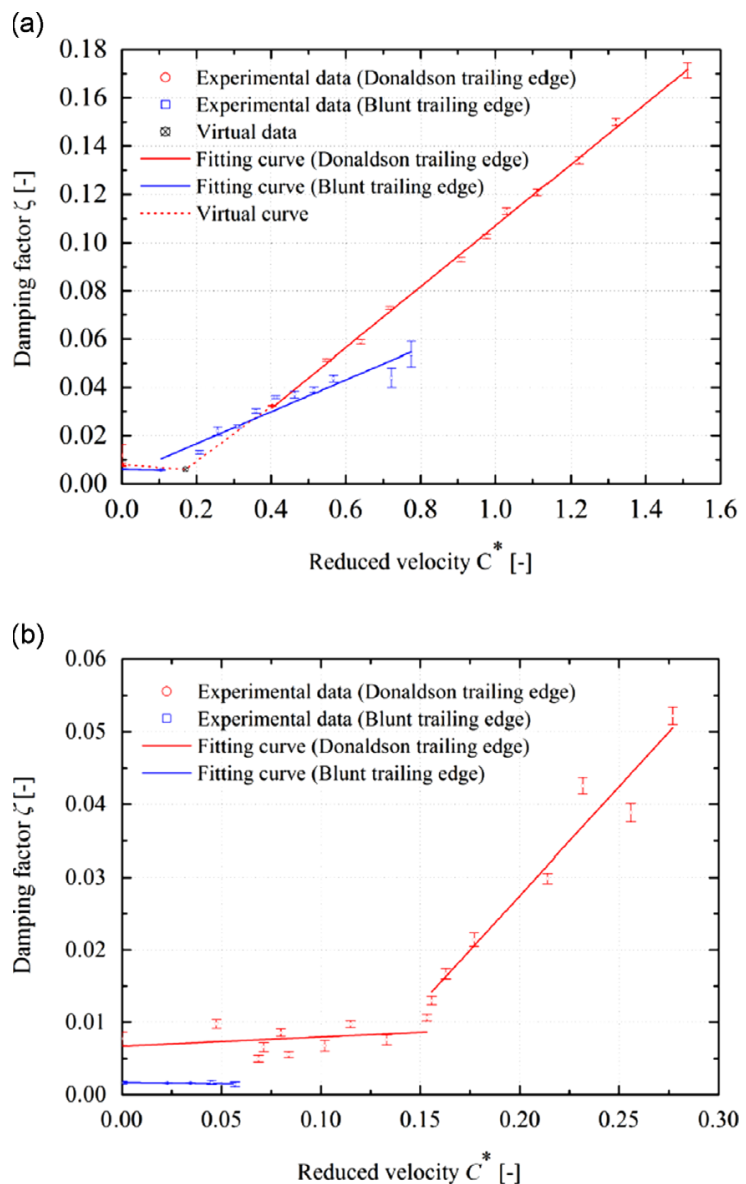

Fig. 8. The damping factors of the first bending mode (a) and the first torsion mode (b) vs reduced velocity.

required to excite the hydrofoil at its 1 st bending mode ( $C^{*} \sim 0.15$ ). According to the above deduction, we prolong the fitting curve near to $C^{*}=0.15$, which is defined as virtual point in Fig. 8(a). And the value of hydrodynamic damping for the virtual point should approximate the one in the still water. Such data processing for the Donaldson trailing edge in the blind zone, which refers to the area with no experimental data, seems to make sense comparing to the results of the blunt trailing edge.

We may observe in Fig. 8 that the hydrodynamic damping is at least 3 times larger for the bending mode than for the torsion mode. We also notice that the damping with the Donaldson trailing edge is significantly larger than with the blunt trailing edge for the torsion mode. This observation is also valid for the bending mode but only at reduced velocities beyond 0.4 . It should be noticed that the resonance at the torsion mode is the most harmful for the mechanical structure because of the possible occurrence of lockin with large vibration amplitudes aver a wide range of flow velocities. Therefore, it appears that the Donaldson trailing edge outperforms the blunt trailing edge because it produces a larger hydrodynamic damping for the torsion mode.

Future work will include the numerical prediction of the interaction phenomena between the hydrofoil and the surrounding water by different trailing edges. And hydrodynamics damping will be modeled and related to the data presented in this manuscript. These predictions will be compared to the flow field as measured by LDV and PIV results, which can be seen in Zobeiri (2012)'s work.

\section{Conclusion}

The identification of hydrodynamic damping of a 2D NACA0009 hydrofoil with Donaldson trailing edge is carried out on a Naca0009 hydrofoil in the test section of the EPFL High Speed Cavitation Tunnel. A specific measurement system using two piezoelectric patches is developed to provide non-intrusive excitation of the hydrofoil in still and flowing water. Free flow induced vibration and forced frequency-sweep vibration are performed to estimate the natural frequencies and the hydrodynamic damping at different flow velocities. The main conclusion may be summarized as follows:

- When the flow velocity is increased from 0 to $21 \mathrm{~m} / \mathrm{s}$, the free vibration of the hydrofoil, induced by the turbulent wake, shows that the bending mode is always excited and reveals a large resonance at the first torsion mode. Unlike the blunt trailing edge, no lock-in was observed for the tested Donaldson trailing edge hydrofoil. 
- Two different trends for the hydrodynamic damping were observed for both bending and torsion modes. A threshold value of about 0.15 was found for the reduce velocity, below which the hydrodynamic remains almost constant and above which it increases linearly.

- When compared with the blunt trailing edge hydrofoil, the hydrodynamic damping of the Donaldson trailing edge is significantly larger, mainly for the 1 st torsion mode.

\section{Acknowledgments}

The present investigation was carried out in a partnership with Laboratory for Hydraulic Machines (LMH) of Swiss Federal Institute of Technology (EPFL). The authors would like to thank Professor Avellan for giving an opportunity to work in LHM, and also thank National Natural Science Foundation of China (No. 51139007), Doctoral Program of Higher Education of China (No. 0100008110012) and China Scholarship Council for the financial support.

\section{References}

Ausoni, P., Farhat, M., Escaler, X., Egusquiza, E., Avellan, F., 2007. Cavitation influence on Von Karman vortex shedding and induced hydrofoil vibration. ASME Journal of Fluids Engineering 129, 966-976.

Avellan, F., Henry, P., Ryhming, I.L., 1987. A New High Speed Cavitation Tunnel, ASME Winter Annual Meeting, vol. 57. Boston, MA, pp. 49-60.

Blevins, R.D., 1990. Flow-Induced Vibrations. Van Norstrand Reinhold, New York.

Bourgoyne, D.A., Hamel, J.M., Ceccio, Dowling, D.R., 2003. Time-averaged flow over a hydrofoil at high Reynolds number. Journal of Fluid Mechanics 496, $365-404$.

Chaplin, J.R., Subbiah, K., 1998. Hydrodynamic damping of a cylinder in still water and in a transverse current. Applied Ocean Research 20 (4), 251-259. Donaldson, M.E., 1956. Hydraulic turbine runner vibration. Journal of Engineering for Power 78, 1141-1147.

De La Torre, O., Escaler, X., Egusquiza, E., Dreyer, M., Farhat, M., 2012. The effect of cavitation on the natural frequency of a hydrofoil. In: Proceeding of the 8th International Symposium on Cavitation. CAV2012-Submission No. 95, Singapore.

De La Torre, O., Escaler, X., Egusquiza, E., Farhat, M., 2013. Experimental investigation of added mass effects on a hydrofoil under cavitation conditions Journal of Fluids and Structure 39, 173-187.

De La Torre, O., 2013. (Ph.D. thesis). Influence of Cavitation on the Dynamic Response of HydrofoilPolytechnic University of Catalonia, Barcelona, Spain.

Howe, M.S., 1988. The influence of surface rounding on trailing edge noise. Journal of Sound and Vibration 126 (3), $503-523$.

Krentel, D., Nitsche, W., 2013. Investigation of the near and far wake of a bluff airfoil model with trailing edge modifications using time-resolved particle image velocimetry. Experiments in Fluids 727, 203-233.

Kaminer, A.A., Nastenko, N.Ya., 1976. Experimental investigation of hydrodynamic damping during bending oscillations of blade profiles in water flow. Strength of Materials 8 (1), 25-27.

Mosallem, M.M., 2008. Numerical and experimental investigation of beveled trailing edge flow field. Journal of Hydrodynamics 20 (3), $273-279$.

Naudascher, E., Rockwell, D., 2006. Flow Induced Vibrations, an Engineering Guide. Elsevier, New York.

Ruprecht, A., Kirschner, O., Lippold, F., Buntic, I., 2003. Noise reduction in a small Francis turbine caused by vortex shedding at the trailing edge, numerical analysis and field test. In: IAHR 11th International Meeting of the Work Group on the Behaviour of Hydraulic Machinery Under Steady Oscillatory Conditions. Stuttgart, Germany.

Roth, S., Calmon, M., Farhat, M., Muench, C., Huebner, B., Avellan, F., 2009. Hydrodynamic damping identification from an impulse response of a vibration blade. In: 3rd IAHR International Meeting of the Workgroup on Cavitation and Dynamic Problems in Hydraulic Machinery and Systems. Brno, Czech Republic.

Shannon, D.W., 2007. (Ph.D. thesis). Flow Field and Acoustic Measurements of a Blunt Trailing EdgeUniversity of Notre Dame, Notre Dame, Indiana.

Seeley, C., Coutu, A., Monette, C., Nennemann, B., Marmont, H., 2012. Characterization of hydrofoil damping due to fluid-structure interaction using piezocomposite actuators. Smart Materials and Structures 21, 1-9.

Williamson, C.H.K., Govardhan, R., 2004. Vortex-induced vibrations. Annual Review of Fluid Mechanics 36, $413-455$.

Zobeiri, A., Ausoni, P., Avellan, F., Farhat, M., 2012. How oblique trailing edge of a hydrofoil reduces the vortex-induced vibration. Journal of Fluids and Structures 32, 78-89.

Zobeiri, A., 2012. (Ph.D. thesis). Effect of Hydrofoil Trailing Edge Geometry on the Wake DynamicSwiss Federal Institute of Technology Lausanne, Lausanne, Switzerland. 